\title{
Riego por goteo en cultivares de tomate (Solanum lycopersicum L.), Universidad Nacional Agraria, Managua, Nicaragua
}

\section{Irrigation in tomato cultivars (Solanum lycopersicum L.), Universidad Nacional Agraria, Managua, Nicaragua}

\author{
Henry Alberto Duarte-Canales ${ }^{1}$, Álvaro Benavides González ${ }^{2}$ \\ ${ }^{1}$ MSc. en agroecología y desarrollo sostenible / ORCID: https://orcid.org/0000-0003-3515-845X / hduarte@ci.una.edu.ni \\ ${ }^{2} \mathrm{MSc}$. en sistemas integrales de producción en el trópico / ORCID: https://orcid.org/0000-0002-0596-690X/alvaro.benavides@ci.una.edu.ni \\ Universidad Nacional Agraria, Facultad de Agronomía
}

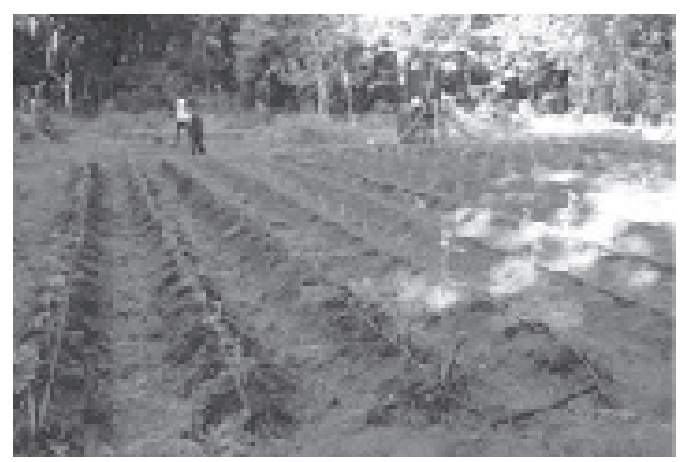

\begin{abstract}
RESUMEN
El tomate (Solanum lycopersicum L.) es una hortaliza importante en Nicaragua por su consumo y comercialización. En el país el cultivo inició en 1940, y actualmente los rendimientos varían entre 12 y $18 \mathrm{t} \mathrm{ha}^{-1}$. Debido a los constantes cambios en el régimen de lluvia, se han desarrollado tecnologías que permiten hacer uso eficiente del agua, como es el riego por goteo. El objetivo de esta investigación fue estudiar tres láminas de riego $\left(\mathrm{T}_{1}=1.5, \mathrm{~T}_{2}=2.0 \mathrm{y} \mathrm{T}_{3}=2.5\right.$ litros planta $^{-1}$ día $^{-1}$ ) en tres cultivares de tomate (Shanty, UC-82 y Butero) en condiciones edafoclimáticas de Managua, Nicaragua. El ensayo se estableció en un diseño de Bloques Completos al Azar (BCA) con arreglo en franjas. Las variables fueron objeto a un análisis de varianza y separación de medias por diferencias mínimas significativas con $p=0.05$. El diámetro polar, diámetro ecuatorial y volumen fueron afectadas significativamente por los dos factores independientes. En la variable rendimiento se encontró diferencias estadísticas en el factor láminas de riego, con mayores promedios 2.0 litros planta ${ }^{-1}$ día $^{-1}\left(11875 \mathrm{~kg} \mathrm{ha}^{-1}\right)$ y 2.5 litros planta ${ }^{-1}$ día $^{-1}(10$ $\left.970 \mathrm{~kg} \mathrm{ha}^{-1}\right)$. Los cultivares que presentaron los mayores promedios fueron Shanty (11 $805 \mathrm{~kg} \mathrm{ha}^{-1}$ ) y Butero con $10945 \mathrm{~kg} \mathrm{ha}^{-1}$. La lámina de riego de 1.5 litros planta ${ }^{-1}$ día $^{-1}$ presentó la mayor pérdida con un coeficiente de cultivo de 3.17, al igual que el cultivar Shanty (3.28); el coeficiente de uniformidad calculado fue del $98.28 \%$ catalogado como excelente.
\end{abstract}

Palabras clave: diámetro polar y ecuatorial, uso eficiente del agua, coeficiente de rendimiento, coeficiente de uniformidad, cultivares.

\begin{abstract}
Tomato (Solanum lycopersicum L.) is an important vegetable in Nicaragua for its consumption and marketing. In Nicaragua, the cultivation of tomato began in 1940, and currently the yields vary between 12 and $18 \mathrm{t} \mathrm{ha}^{-1}$. Due to the constant changes in the rain regime, technologies have been developed that allow efficient use of water, such as drip irrigation. The objective of this research was to study three irrigation sheets $\left(\mathrm{T}_{1}=1.5, \mathrm{~T}_{2}=2.0\right.$ and $\mathrm{T}_{3}=2.5$ liters plant $^{-1}$ day $^{-1}$ ) in three tomato cultivars (Shanty, UC-82 and Butero) in soils conditions of Managua, Nicaragua. The trial was setted up in a Full Random Block (BCA) design with stripe arrangement. The variables were subjected to an analysis of variance and separation of means by minimum significant differences with $p=0.05$. Polar diameter, equatorial diameter, and volume were significantly affected by the two independent factors. In the yield variable, statistical differences were found in the factor of irrigation sheets, with higher averages of 2.0 liters plant ${ }^{-1}$ day $^{-1}\left(11875 \mathrm{~kg} \mathrm{ha}^{-1}\right)$ and 2.5 liters plant ${ }^{-1}$ day $^{-1}\left(10970 \mathrm{~kg} \mathrm{ha}^{-1}\right)$. The cultivars that presented the highest averages were Shanty (11 $\left.805 \mathrm{~kg} \mathrm{ha}^{-1}\right)$ and Butero with $10945 \mathrm{~kg} \mathrm{ha}^{-1}$. The irrigation sheet of 1.5 liters plant ${ }^{-1}$ day $^{-1}$ presented the highest loss with a cultivation coefficient of 3.17 like the cultivar Shanty (3.28); the calculated uniformity coefficient was $98.28 \%$ classified as excellent.
\end{abstract}

Keywords: Polar and equatorial diameter, efficient use of water, yield coefficient, uniformity coefficient, cultivars.
Recibido: 10 de diciembre del 2019 Aceptado: 29 de abril del 2020

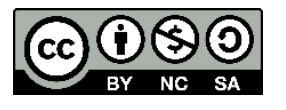

Los artículos de la revista La Calera de la Universidad Nacional Agraria, Nicaragua, se comparten bajo términos de la licencia Creative Commons: Reconocimiento, No Comercial, Compartir Igual. Las autorizaciones adicionales a las aquí delimitadas se pueden obtener en el correo edgardo.jimenez@ci.una.edu.ni 


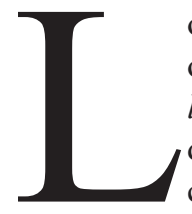
os hábitos de consumo y variedad de productos obtenidos convierten al tomate (Solanum lycopersicum L.) en una industria de gran dinamismo y niveles crecientes de consumo. Este cultivo se adapta en particular a productores que tienen disponibilidad de mano de obra familiar y presentan una débil articulación comercial; aunque pueden presentar ciertas limitaciones productivas por suelos degradados y restricciones en disponibilidad de fuentes de agua (García, Martínez, Avendaño, Padilla y Izquierdo, 2009). Esta hortaliza presenta ventajas económicas y nutritivas; catalogada como una fuente de vitaminas A y $\mathrm{C}$ que ayudan a corregir las deficiencias de las mismas. En Nicaragua las hortalizas son importantes por su potencial económico y su demanda alimenticia. Su producción se adapta bien a las condiciones agroclimáticas de los trópicos, particularmente bajo riego y en zonas altas con marcadas períodos secos [Instituto Nicaragüense de Tecnologías Agropecuaria (INTA, 2004)].

Considerando la importancia de este rubro y los constantes cambios en el régimen de lluvias, se han desarrollado tecnologías que permiten hacer uso eficiente del agua, como es el riego por goteo, este es un método de irrigación utilizado en diferentes cultivos, lo que permite un mejor aprovechamiento del agua y fertilizantes, tanto orgánicos como químicos. El agua aplicada por este método de riego se infiltra hacia las raíces de las plantas regando directamente la zona de influencia del sistema radical a través de un sistema de tuberías y emisores (Duarte y Ruíz, 2010; Erazo, 2015).

En el cultivo de tomate es importante el manejo de agua, depende el éxito o fracaso del mismo (Wilches-Rojas, Álvarez-Herrera y Balaguera-López, 2008). La principal problemática del sector agrícola es el uso deficiente y mal control del agua de riego que utilizan para sus cultivos, por ello surge la necesidad de realizar estudios previos sobre las necesidades hídricas de este cultivo, evaluando láminas de riego para contribuir que los productores le den un uso adecuado al recurso hídrico.

El consumo diario de agua por planta adulta de tomate es de aproximadamente 1.5 a 2 litros planta $^{-1}$ día $^{-1}$, la cual varía dependiendo de la zona, la variedad utilizada, las condiciones climáticas, la época del año y tipo de suelo. En general con el riego por goteo se aplica entre tres a cuatro litros de agua $\mathrm{m}^{2}$ por día ${ }^{-1}$ (Chemonics International Inc, 2008).

\section{MATERIALES Y MÉTODOS}

El estudio se desarrolló en el departamento de Managua, Nicaragua en el área experimental de la Facultad de Agronomía de la Universidad Nacional Agraria (UNA), ubicada en el km 12.5 Carretera Norte. Este se encuentra a $12^{\circ} 08^{\prime} 36^{\prime \prime}$ latitud
Norte y $86^{\circ} 9^{\prime} 49^{\prime \prime}$ de longitud Oeste, a una altitud de $56 \mathrm{msnm}$. El suelo presenta un $\mathrm{pH}$ de 8.03 , con pendiente entre 0 a $2 \%$ (Martínez y Meza, 2011). Según Cuadra y García (2016), el tipo de suelo donde se estableció el experimento en el periodo de noviembre a mayo del 2015 al 2017, pertenece a la serie La Calera, del orden Inceptisol, de color negro y pobremente drenado debido a que la permeabilidad es lenta, textura franco arenoso (Arcilla 11.2\%, Limo 28\% y Arena 60.8\%).

Diseño y variables evaluados. El experimento fue establecido en un diseño de Bloques Completos al Azar (BCA) con arreglo en franjas y tres réplicas, cada una de las réplicas estuvo constituida por tres parcelas, cuyas dimensiones fueron $4 \mathrm{~m}$ x $5 \mathrm{~m}\left(180 \mathrm{~m}^{2}\right.$ en total $)$. La cinta de goteo tenía $16 \mathrm{~mm}$ de diámetro, espaciamiento de $40 \mathrm{~cm}$ entre emisores con un gasto de 1.5 litros por hora y una presión de trabajo de $150 \mathrm{kPa}$. Los tratamientos en estudios variaron de acuerdo a las fases fenológicas del cultivo iniciando con (1.5 1 planta $^{-1}$ día $^{-1}, 2.01$ planta $^{-1}$ día $^{-1}$ y 2.51 planta $^{-1}$ día $^{-1}$ ), fase intermedia (3 1 planta $^{-1}$ día $^{-1}, 4.01$ planta $^{-1}$ día $^{-1}$ y 51 planta $^{-1}$ día $^{-1}$ ), fase final (1.5 1 planta $^{-1}$ día $^{-1}, 2.01$ planta $^{-1}$ día $^{-1}$ y 2.5 1 planta $^{-1}$ día $\left.^{-1}\right)$ y tres cultivares de tomate: Shanty, UC-82 y Butero. Las variables fueron: diámetro polar y ecuatorial $(\mathrm{cm})$, volumen del fruto $\left(\mathrm{mm}^{3}\right)$ y rendimiento $\left(\mathrm{kg} \mathrm{ha}{ }^{-1}\right)$.

Arreglo de láminas de riego. La medición del contenido de agua del suelo tiene fundamental importancia para el riego, con el fin de establecer la frecuencia de riego (cuando regar) y la lámina a reponer en el suelo durante el riego (cuanto regar). Estos aspectos están en dependencia de la capacidad de almacenamiento del agua y del peso específico aparente del suelo, así como de la profundidad de raíces o zona de absorción. La cantidad de agua aplicada se expresó en términos de carga o lámina en mm (Valverde, 2000). La fórmula para la determinación de la lámina de agua es la siguiente:

$L a=(C C-P M P / 10) * D a * P r$

Donde:

La: Lámina de agua en ( $\mathrm{mm})$.

Cc: Contenido de humedad del suelo a capacidad de campo (\% de masa)

PMP: Contenido de humedad del suelo a punto de marchitez (\% de masa)

Da: Densidad aparente del suelo $\left(\mathrm{gr}^{3} \mathrm{~cm}^{3}\right)$

Pr: Profundidad de raíces del cultivo $(\mathrm{cm})$

\section{Variables de riego}

Coeficiente de rendimiento (Ky). El rendimiento de un cultivo está estrechamente ligado al agua que este evapotranspirada. Conocer la relación agua-rendimiento 
(Ky) es de gran importancia para planificar el riego y para proyectar el rendimiento de los cultivos (Valle y Velásquez, 2019).

Este cálculo se realizó al final de la cosecha por medio de la fórmula:

$$
k y=\frac{\left(1 \frac{y r}{y m}\right)}{\left(1 \frac{\text { Evptr }}{\text { Evtpm }}\right)}
$$

Donde:

Yr: Rendimiento real de la cosecha

Ym: Rendimiento máximo

Evtpm: Evapotranspiración máxima

Evtpr: Evapotranspiración real

Coeficiente de uniformidad (CU). Fue determinada mediante el uso de un recipiente y una probeta de $100 \mathrm{ml}$, para ello se seleccionaron tres emisores ubicados en los laterales críticos. Transcurrida una hora se procedió a la medición del agua recolectada en los recipientes colectores, Para proceder a los cálculos por el método del cuarto menor propuesta por el Instituto de Protección Agropecuario (INIAURURI, 2010), los datos transformados en litros por hora para su registro y posterior análisis.

$\mathrm{CUC}=\frac{q_{25 \%}}{q_{n}} \times 100$

Donde:

CUC: Coeficiente de uniformidad del Caudal (\%).

$\mathrm{q}_{25 \%}$ : Caudal promedio (litros horas ${ }^{-1}$ ) de los cuatro emisores de menor caudal

$\mathrm{q}_{\mathrm{n}}$ : Caudal de todos los emisores (litros horas ${ }^{-1}$ ).
Variables agronómicas y análisis de la información. En la cosecha se midió el diámetro polar y ecuatorial $(\mathrm{cm})$ en 10 frutos de cada tratamiento; volumen de fruto $\left(\mathrm{mm}^{3}\right)$ en un beaker de $1000 \mathrm{ml}$ con volumen de agua de $300 \mathrm{ml} \mathrm{y}$ medida el agua desplazada en $\mathrm{cm}^{3}$, así como el rendimiento en $\mathrm{kg} \mathrm{ha}^{-1}$.

La información biométrica fue sometida a un análisis de varianza y categorización estadística (DMS, $p=0.05$ ) utilizando el software R (R Team, 2019).

\section{RESULTADOS Y DISCUSIÓN}

Variables de fruto. El diámetro polar y ecuatorial del fruto varía según el tamaño y la forma del mismo. El tamaño del fruto es variable según el material genético y alcanza diámetros variables (Mayorga, 2004).

El mayor diámetro polar del fruto se midió en la lámina de 1.5 litros planta ${ }^{-1}$ día $^{-1}$ y 2.0 litros planta ${ }^{-1}$ día $^{-1}$, en cuanto a los cultivares el que presentó el mayor promedio fue Shanty, los valores obtenidos coinciden con los alcanzados por Gómez y Herrera (2014); Olivas y Salgado (2013).

El diámetro ecuatorial de los frutos con las mayores medias se obtuvo en las láminas de riego y cultivares, 1.5 litros planta ${ }^{-1}$ día $^{-1}$ y 2.0 litros planta ${ }^{-1}$ día $^{-1}$, Shanty y UC-82 (Cuadro 1). Estos resultados son superiores a los reportados por López y Coleman (2016) con promedios de 2.2 y $3.1 \mathrm{~cm}$.

Los mayores volúmenes del fruto lo obtuvieron Shanty y UC-82. Los resultados fueron superiores a los reportados por López y Coleman (2016) con valores de 28.39 a $51.17 \mathrm{~mm}^{3}$.

Los mayores rendimientos se obtuvieron con las láminas de 2.0 litros planta ${ }^{-1}$ día $^{-1}$ y 2.5 litros planta ${ }^{-1}$ día $^{-1}$. Para el caso de los cultivares los mayores promedios se registran con Shanty y Butero. Estos resultados son superiores a los obtenidos por Cuadra y García (2016), con un promedio de $9676.7 \mathrm{~kg} \mathrm{ha}^{-1}$ con la aplicación de la misma lámina de riego 2.0 litros planta ${ }^{-1}$ día $^{-1}$. El Ministerio Agropecuario y Forestal (MAGFOR, 2007) y la Organización de las
Volumen total de agua aplicada. El volumen total de agua aplicada en todo el ciclo del cultivo se calculó de acuerdo a las tres láminas de riego utilizadas, en todos sus estados de desarrollo.
Cuadro 1. Valores medios en variables de fruto del cultivo de tomate estimados en los efectos principales

\begin{tabular}{|c|c|c|c|c|c|}
\hline Factores & Nivel & $\begin{array}{c}\text { Diámetro polar } \\
\mathrm{cm}\end{array}$ & $\begin{array}{c}\text { Diámetro ecuatorial } \\
\mathrm{cm}\end{array}$ & $\begin{array}{c}\text { Volumen de fruto } \\
\mathrm{mm}^{3}\end{array}$ & $\begin{array}{c}\text { Rendimiento } \\
\mathrm{kg} \mathrm{ha}^{-1}\end{array}$ \\
\hline \multirow[t]{3}{*}{ Láminas de riego } & 1.5 litros planta ${ }^{-1} \mathrm{dia}^{-1}$ & $5.15 \mathrm{a}$ & $4.31 \mathrm{a}$ & $57.87 \mathrm{a}$ & $9388 \mathrm{~b}$ \\
\hline & 2.0 litros planta ${ }^{-1}$ día $^{-1}$ & $5.17 \mathrm{a}$ & $4.30 \mathrm{a}$ & $61.77 \mathrm{a}$ & $11875 \mathrm{a}$ \\
\hline & 2.5 litros planta ${ }^{-1}$ día $^{-1}$ & $4.51 \mathrm{~b}$ & $3.97 \mathrm{~b}$ & $59.82 \mathrm{a}$ & $10970 \mathrm{ab}$ \\
\hline \multirow[t]{5}{*}{ Cultivares } & Shanty & $5.85 \mathrm{a}$ & $4.45 \mathrm{a}$ & $65.97 \mathrm{a}$ & 11805 a \\
\hline & UC-82 & $4.90 \mathrm{~b}$ & $4.29 \mathrm{a}$ & $65.77 \mathrm{a}$ & 9557 b \\
\hline & Butero & $4.58 \mathrm{c}$ & $4.00 \mathrm{~b}$ & $49.50 \mathrm{~b}$ & $10945 \mathrm{ab}$ \\
\hline & CV (\%) & 8.36 & 7.91 & 9.75 & 8.61 \\
\hline & $\mathrm{R}^{2}$ & 0.70 & 0.80 & 0.78 & 0.75 \\
\hline
\end{tabular}

Letras similares en columnas indican no significación estadística (DMS, $p>0.05$ ). 
Naciones Unidas para la Alimentación y la Agricultura (FAO, 2012) mencionan una producción promedio de 14 $633.96 \mathrm{~kg} \mathrm{ha}^{-1}$.

El volumen promedio de agua aplicado en todo el ciclo del cultivo se calculó con respecto a las láminas de riego en los diferentes estados de desarrollo del cultivo. En el Cuadro 2, al hacer la sumatoria del agua aplicada, se obtiene que, a los 120 días de desarrollo del cultivo, el volumen total de agua fue de $138.88 \mathrm{~m}^{3}$.

Cuadro 2. Volumen promedio de agua utilizada en las láminas de riego en litros evaluadas en los cultivares Shanty, UC-82 y Butero para un ciclo 120 días en el campo

\begin{tabular}{|c|c|c|c|c|c|}
\hline Factor & Nivel & $\begin{array}{c}\text { Etapa inicial } \\
(0-45 \mathrm{ddt})\end{array}$ & $\begin{array}{l}\text { Etapa media } \\
(45-105 \mathrm{ddt})\end{array}$ & $\begin{array}{c}\text { Etapa final } \\
(105-120 \mathrm{ddt})\end{array}$ & $\begin{array}{c}\text { Agua aplicada } \\
\left(\mathrm{m}^{3}\right)\end{array}$ \\
\hline \multirow[t]{3}{*}{ Láminas de riego } & 1.5 litros planta ${ }^{-1}$ día $^{-1}$ & 12.83 & 17.10 & 4.28 & 34.20 \\
\hline & 2.0 litros planta ${ }^{-1}$ día $^{-1}$ & 17.10 & 22.80 & 5.70 & 47.60 \\
\hline & 2.5 litros planta ${ }^{-1}$ día $^{-1}$ & 21.38 & 28.50 & 7.13 & 57.00 \\
\hline
\end{tabular}

Ddt=Días después del trasplante.

Coeficiente de rendimiento del cultivo $\mathbf{K y}$. Considerando los factores evaluados (láminas de riego y cultivares de tomate), la lámina de riego con menor pérdida en el rendimiento fue de 2.5 litros planta ${ }^{-1}$ día $^{-1}$ con un Ky de 2.26 registrando mayor pérdida la lámina de 1.5 litros planta ${ }^{-1}$ día $^{-1}$ con un Ky de 3.17 (Figura 1). El cultivar que presentó la mayor pérdida fue Shanty (3.28) y con un menor Ky Butero (2.60). Esta diferencia se debe a la cantidad de agua aplicada.
El rendimiento de cultivo con mayor valor de Ky, sufrirá mayor pérdida que el cultivo con menor valor de Ky (Doorenbos y Kassam, 1980). Asimismo, la producción de los cultivos depende de muchos factores, entre ellos las variedades, fertilización, control de plagas y riego, de todos ellos el agua es uno de los más limitantes y su control es fundamental para el éxito en la producción agrícola (Santiago y Borrego,1998; Leskovar, 2001; Salazar-Antón y Hernández, 2013).

Coeficiente de uniformidad. El coeficiente de uniformidad calculado fue del $98.28 \%$ catalogándose como excelente. La topografía, las variaciones propias entre emisores por manufactura y las pérdidas de carga en la red no afectaron de manera significativa la uniformidad de descarga. Mediante las observaciones realizadas al sistema de riego, en cada operación, no ocurrió obstrucción de emisores durante el ensayo.

Pizarro (1996) expresa que, en los sistemas de riego localizado, el coeficiente de uniformidad debe superar el $90 \%$. utilizando el método del cuarto menor. Asimismo, Cun, Puig y Morales (2009), deducen que un coeficiente de uniformidad de $98 \%$ está es un rango de excelente, siempre y cuando no se vea afectado por las limitaciones en las descargas del sistema.

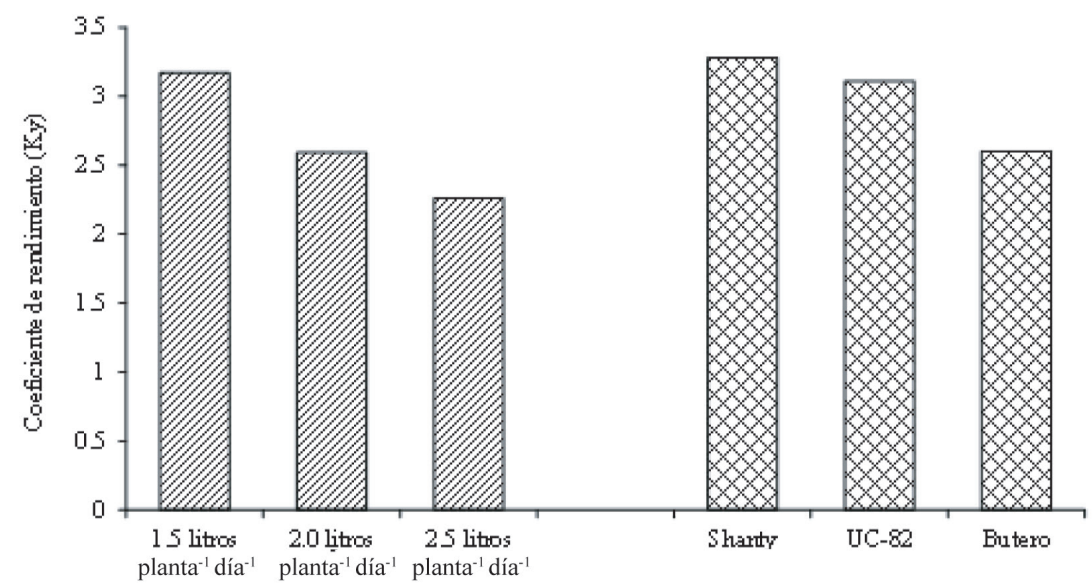

Figura 1. Coeficientes de rendimiento $(\mathrm{Ky})$ obtenidos según láminas de riego y cultivares de tomate.

\section{CONCLUSIONES}

Las variables diámetro polar y ecuatorial fueron afectadas significativamente por las láminas de riego 1.5 y 2.0 litros planta ${ }^{-1}$ día $^{-1}$. Los mayores rendimientos se obtuvieron en la variedad Shanty con la lámina de riego de 2.0 litros planta ${ }^{-1}$ día $^{-1}$ manejando un gasto de $47.60 \mathrm{~m}^{3}$ de agua en todo el siclo del cultivo. En cuanto al coeficiente de rendimiento (Ky) los tratamientos con menores pérdidas fueron 2.5 litros planta $^{-1}$ día $^{-1}$ y con el cultivar Butero. Asimismo, el sistema resultó altamente uniforme en cada uno de los tratamientos.

Este factor Ky en los cultivos es esencial para la planificación, diseño y operación de los proyectos de riego, ya que cuantifican el agua en términos de rendimiento del cultivo y producción total para la superficie cultivada. 


\section{REFERENCIAS BIBLIOGRÁFICAS}

Chemonics International Inc, (2008). Programa de diversificación hortícola, Proyecto de desarrollo de la cadena de valor y conglomerado agricola. Cultivo de tomate (Lycopersicum esculemtum Mill): Manual de cultivo de tomate.

Cuadra Aguilera, F., y García Ramos, D. (2016). Evaluación de tres láminas de riego por goteo y tres dosis de biofertilizante, en el cultivo de tomate (Lycopersicum esculentum Mill) variedad Shanty en condiciones edafoclimáticas de Managua, Universidad Nacional Agraria, Managua, Nicaragua. (Tesis de grado) Universidad Nacional Agraria, Managua, Nicaragua. https://repositorio.una.edu.ni/3434/1/tnf06c961.pdf.

Doorenbos, J., y Kassam, A. (1980). Efectos del agua sobre rendimiento de los cultivos. Estudios FAO: Riego y Drenaje. Ed. FAO. Roma, Italia. p. 41-213.

Duarte, H. A., y Ruiz, M. (2010). Efecto de tres láminas de riego y tres dosis de aplicación de biofertilizantes en el cultivo orgánico de fresa (Fragaria spp.) cv. Festival en El Castillito, Las Sabanas, Madriz. (Tesis de grado). Universidad Nacional Agraria, Mangua, Nicaragua. https://cenida.una.edu.ni/Tesis/tnf04d812.pdf.

Erazo Yépez, L. E. (2015). Diseño de un sistema de riego para la hacienda San Antonio, ubicada en la parroquia Machachi, cantón Mejía, provincia de Pichincha. (Tesis de grado) Quito: USFQ]. http://repositorio.usfq.edu.ec/handle/23000/4996

Organización de las Naciones Unidas para la Alimentación y la Agricultura. (2012). Nicaragua: La batalla por los rendimientos agropecuarios. Dirección de estadísticas. http://www.fao.org/3/a-i3028s.pdf.

García, M. L. V., Martínez, A. N., Avendaño, M. C., y Padilla H, Izquierdo. (2009) Acción de oligosacáridos en el rendimiento y calidad detomate. Rev. Fitotec. Mex. 32:(4), 295-301.

Gómez Peralta, D., y Herrera Fuente, E. (2014). Comportamiento agronómico de 12 cultivares de tomate (Lycopersicum esculentum Mill) en condiciones de campo en Tisma, Masaya y en casa malla, en el CEVT Las Mercedes, UNA. (Tesis de grado). Universidad Nacional Agraria, Managua, Nicaragua. https://lacalera.una.edu.ni/index.php/CALERA/article/ view/251/246.

Instituto de Protección Agropecuaria. (2010). Determinación del coeficiente de uniformidad de riego. Ed. Centro de Investigación Especializado en Agricultura del Desierto y Altiplano (CIE). Arica, CL. 2 p.

Instituto Nicaragüense de Tecnología Agropecuaria. (2004). Manejo integrado de plagas: Cultivo del tomate guía MIP. 64 p.

Leskovar, D. I. (2001). Producción y ecofisiología del transplante hortícola. Texas A y University.

López, Elieth, P., y Coleman Beer, E. (2016). Efecto de tres láminas de riego por goteo y tres distancias de siembra en el cultivo de tomate (Solanum lycopersicum L.) cv. UC-82. (Tesis de grado). Universidad Nacional Agraria, Managua, Nicaragua. https://repositorio.una.edu.ni/3415/1/tnf061864.pdf

Ministerio Agropecuario y Forestal. (2007). Área cosechada, rendimientos y producción de hortalizas a nivel nacional. ciclos agrícolas del 1999-2005. Managua, Nicaragua. Estudio Preliminar. https://www.enriquebolanos.org/media/informe/ MAGFOR\%202002\%20-\%202006.pdf

Martínez Nuñez, A. D., y Meza Granados, N. A. (2011). Evaluacion de riego y biofertilizante sobre seis poblaciones de tomate silvestre (Lycopersicum spp.) colectado en la Reserva de Recursos Genéticos de Apacunca (RRGA), Chinandega. (Tesis de grado). Universidad Nacional Agraria, Managua, Nicaragua. https://repositorio.una.edu.ni/2156/1/tnf06m385.pdf

Mayorga Suchite, A. S. (2004). Evaluación agronómica de ocho hibridos de tomate (Lycopersicum esculentum Mill) en dos localidades de Zacapa. Chiquimula, GA. USAC. http://cunori.edu.gt/descargas/EVALUACIN_AGRONOMICA_DE_ OCHO_HIBRIDOS_DE_TOMATE_EN_DOS_LOCALIDADES_DE_ZACAPA.pdf.

Olivas, L. y Salgado, L. (2013). Evaluación de rendimiento y comportamiento agronómico de siete genotipos de tomate (Lycopersicum esculentum, Mill.) bajo sistema de casa malla en el centro experimental Las Mercedes. (Tesis de grado). Universidad Nacional Agraria, Managua, Nicaragua. https://repositorio.una.edu.ni/2205/1/tnf30o48.pdf

Pizarro, F. (1996). Riego localizado de alta frecuencia Goteo Microaspersión y exudación. Editorial, Mundi - Prensa libro. 471 p.

R Core Team (2019). R: A language and environment for statistical computing. R Foundation for Statistical Computing, Vienna, Austria. https://www.R-project.org/.

Cun González, R., Puig Estrada, O., y Morales Gómez, C. (2009). Comportamiento del coeficiente de uniformidad del riego por microaspersión en condiciones deorganopónicos y huerto intensivo. Revista Ciencias Técnicas Agropecuarias, 18(3). https:// www.redalyc.org/pdf/932/93215944007.pdf

Salazar-Antón, W. y Guzmán-Hernández, T. D. J. (2013). Nematodos fitoparásitos asociados al tomate en la zona occidental de Nicaragua. Agronomía Mesoamericana, 24(1), 27-36 p.

Santiago, J., y Borrego, F. (1998). Evaluación de tomate (Lycopersicon esculentum, Mill) en invernadero: criterios fenológicos y fisiológicos. Agronomía mesoamericana. p. 59-65. 
Valle López, H. S. y Velásquez, M. L. (2019). Evaluación de fertilizantes sintéticos y orgánica en el cultivo de maiz (Zea mays L.) variedad NB-6 bajo riego por microaspersión en la Finca El Plantel, 2017-2018. (Tesis de grado). Universidad Nacional Agraria, Managua, Nicaragua. https://repositorio.una.edu.ni/3833/1/tnf04v181f.pdf

Valverde Conejo, J. C. (2000). Riego y drenaje. 1. Reimp. 1. Ed. San José, C.R.: UNED, 2000. 248 p.

Wilches-Rojas, F. A., Álvarez-Herrera, J. G., y Balaguera-López, H. E. (2008). Alveolus size and different watering levels in the production of tomato seedlings. Revista UDCA Actualidad \& Divulgación Cientifica, 11(2), 141-151. http://www. scielo.org.co/scielo.php?script=sci_arttext\&pid=S0123-42262008000200015 